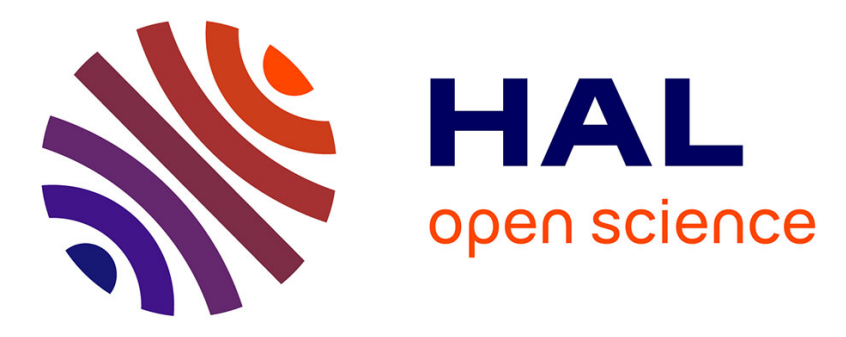

\title{
Interactive Ultrasonic Field Simulation For Non-Destructive Testing
}

Jason Lambert, Hamza Chouh, Gilles Rougeron, Vincent Bergeaud, Sylvain

Chatillon, Lionel Lacassagne, Jean-Claude Iehl, Jean-Philippe Farrugia, Victor Ostromoukhov

\section{To cite this version:}

Jason Lambert, Hamza Chouh, Gilles Rougeron, Vincent Bergeaud, Sylvain Chatillon, et al.. Interactive Ultrasonic Field Simulation For Non-Destructive Testing. 25th Eurographics Symposium on Rendering, Jun 2014, Lyon, France. , 33 (2), 2014, 10.1117/12.2182840 . hal-01093294

\section{HAL Id: hal-01093294 \\ https://inria.hal.science/hal-01093294}

Submitted on 10 Dec 2014

HAL is a multi-disciplinary open access archive for the deposit and dissemination of scientific research documents, whether they are published or not. The documents may come from teaching and research institutions in France or abroad, or from public or private research centers.
L'archive ouverte pluridisciplinaire HAL, est destinée au dépôt et à la diffusion de documents scientifiques de niveau recherche, publiés ou non, émanant des établissements d'enseignement et de recherche français ou étrangers, des laboratoires publics ou privés. 


\title{
Interactive Ultrasonic Field Simulation For Non-Destructive Testing
}

\author{
J. Lambert ${ }^{1}$, H. Chouh ${ }^{1}$, G. Rougeron ${ }^{1}$, V. Bergeaud ${ }^{1}$, S. Chatillon ${ }^{1}$, L. Lacassagne ${ }^{2}$, J.C. Iehl ${ }^{3}$, J.P. Farrugia ${ }^{3}$ and V. Ostromoukhov $^{3}$ \\ ${ }^{1}$ CEA-LIST, CEA Saclay, France \\ ${ }^{2}$ LRI, UMR 8623, Univ. Paris Sud 11, Orsay, France \\ ${ }^{3}$ LIRIS, UMR 5205, Univ. Lyon 1, Villeurbanne, France
}

\begin{abstract}
In Non Destructive Testing domain there a is a need for interactive simulation tools. This poster presents such a tool which computes up to 20 ultrasonic field images per seconds for simple configurations on a high-end CPU. Extensions to handle more complex configurations are also presented.

Categories and Subject Descriptors (according to ACM CCS): F.1.2 [Computation by Abstract Devices]: Modes of Computation-Interactive and reactive computation
\end{abstract}

\section{Introduction}

Inspection simulation is used in a lot of non-destructive testing (NDT) applications in industry. The aim of Civa Software is to provide fast and easy to interpret NDT simulations and reconstructions. In this context, this poster presents a new tool for the interactive simulation of ultrasonic fields.

\section{Ultrasonic Field Simulation Model}

An ultrasonic field simulation computes the field radiated by a probe (immersed or in contact, mono-element or phased array) in an inspected specimen. It is generally used to choose the best suited characteristics of a probe for a given control.

Ultrasonic waves propagation into solids involves complex physical phenomena. Among them, the following ones can be highlighted. Different types of volumic waves can propagate at different celerities. At each surfacic interface they can be specularly reflected or refracted, with possibly wave type conversion. Specimen can be homegeneous or inhomogeneous. Materials can be isotropic or anisotropic. ${ }^{\dagger}$ In Civa Software, an ultrasonic field simulation consists in three steps [Gen03]:

1. performing pencil tracing by computing for each pencil on one hand an axial ray that starts from a source point

\footnotetext{
$\dagger$ Anisotropy implies the waves celerity depends on the propagation direction.
}

on the probe and reaches the discretized field area, and on the other hand the following parameters : amplitude (via a divergence factor and the multiplication of Fresnel coefficients), time of flight and duration.

2. delaying and accumulating pencil contributions on a vectorial displacement impulse response $: \overrightarrow{I R_{D}(t)} \in C^{3}$ according to the delay laws used for the probe,

3. convoluting this response with the probe signal and extracting a max amplitude for each field point which leads to a colored image.

Field simulations in current Civa software can handle very complex configurations at the price of often important computation time (typically from dozens of seconds to hours). A new tool adressing simple configurations has therefore been developped to reach our interactivity goal.

\section{A fast implementation for simple configurations}

A simplified model, restricted to homogeneous isotropic specimens with planar boundaries has been developped. Single refracted ray paths are computed through a 1D-Newton equation resolution ( [WZHB09]). A supplementary reflection without conversion can easily be added. Pencil parameters are computed directly through analytic formulae.

A first reference, OpenMP parallelized, scalar, single precision implementation in three steps, using Intel MKL FFT, has been coded on CPU. It has then been further accelerated. Using Boost.SIMD library, steps 1. and 3. have been 
vectorized. Morevover, a single step algorithm avoiding the unnecessary storage of temporary data and providing more work for each thread has been settled. The resulting optimized code scales well on a $2 \times 12$ cores CPU. Its overall performance is around $3.5 \mathrm{x}$ faster than previous reference implementation on a set of representative configurations. For the simplest ones, it reaches $20 \mathrm{fps}$.

This implementation shall be tested on other harwdares (CPUs with AVX/AVX2 registers and Intel MIC). An optimized GPU Cuda based code shall be implemented.

\section{Towards more complexity}

Previous implementation can be functionnaly extended in order to take into account :

- non planar surfaces, which raises the complexity of nonlinear equations to solve,

- the length of ray paths with a second reflection or refraction on the specimen boundaries with possibly wave conversion, which leads to solve a system of equations

- specimen with many boundaries, which means some heuristic must be used in order to avoid testing all possible combinations of surfaces for a given path type.

[JM12] might be used to find neighbor paths starting from a known specular path.

But as these extensions remains limited, for even more complex configurations, i.e. :

- specimen with any geometry (non-planar surfaces or 3D triangle mesh)

- inhomogeneous specimen with many different materials

- anisotropic materials

- paths of any length

another method based on fast ray tracing has to be developped. Early experimentations showed that an ultrasonic raytracer based on Intel Embree on CPU or Nvida OptiX on GPU should allow us to reach the necessary level of performance required by interactivity (at least via progressive computation). Furthermore a geometrical method has been settled to trace rays in anistropic materials much faster than Civa analytic method. Fast methods for Fresnel coefficient and divergence factor computation might be worked out in order to compute full pencil parameters.

Relying on these fast tools, the main algorithmic effort shall concern the pencil computation step as for complex configurations it represents by far the major part of computation time. Two different ideas shall be explored :

1. Pencil computation in two sub-steps :

- A geometrical sub-step = For each field point and each path type, find solid angles through which the probe surface is seen via a reverse ray tracer guided by an adaptive algorithm (an optimization algorithm aiming at minimizing the distance of rays to the probe surface, or AD-Frustum [CLT* 08])

- A physical sub-step = For each field point, each path type and each solid angle, discretize the surface probe into a set of source points and perform pencil tracing starting from the probe.

2. Pencil computation in one step : Perform a MonteCarlo light tracing (or Markov Chain Monte Carlo) with importance sampling, and possibly regularization (like in [KD13]), following ray paths starting from the probe until they reach the field area and contribute to the field points impulse responses.

\section{Perspective and conclusion}

A fast US field simulation tool allowing interactive change of any parameter for simple configurations has been developped on CPU. New hardware shall be tested (CPUs with larger SIMD registers, Intel MIC, and GPUs). The method shall be extended to take into account more complex geometries or longer ray paths. For very complex configurations, in particular those with anisotropic materials, an alternative method using ray tracing is currently being developped. The resulting interactive tools will be integrated in next Civa software commercial release. As different codes developed for different hardware will finally be available, an auto-tuning mechanism will be settled in order to choose automatically the best one for a given ultrasonic field configuration. Another mechanism might be developed to automatically tune computation options in order to keep a satisfactory level of interactivity [GD12]

\section{References}

[CLT*08] Chandak A., Lauterbach C., Taylor M., Ren Z., MANOCHA D.: Ad-frustum: Adaptive frustum tracing for interactive sound propagation. Visualization and Computer Graphics, IEEE Transactions on 14, 6 (Nov 2008), 1707-1722. 2

[GD12] Ganestam P., Doggett M.: Auto-tuning interactive ray tracing using an analytical gpu architecture model. In Proceedings of the 5th Annual Workshop on General Purpose Processing with Graphics Processing Units (New York, NY, USA, 2012), GPGPU-5, ACM, pp. 94-100. 2

[Gen03] Gengembre N.: Pencil method for ultrasonic beam computation. in Proc. of the 5th World Congress on Ultrasonics (2003), 1533-1536. 1

[JM12] JaKob W., MarSchner S.: Manifold exploration: A markov chain monte carlo technique for rendering scenes with difficult specular transport. ACM Trans. Graph. 31, 4 (July 2012), 58:1-58:13. 2

[KD13] Kaplanyan A. S., Dachsbacher C.: Path space regularization for holistic and robust light transport. Computer Graphics Forum (Proc. of Eurographics 2013) 32, 2 (2013), 63 72. 2

[WZhB09] Walter B., Zhao S., Holzschuch N., Bala K.: Single Scattering in Refractive Media with Triangle Mesh Boundaries. ACM Transactions on Graphics 28, 3 (Aug. 2009), 92:1-8. 1 